\title{
An unusual cause of chest pain in a young woman
}

\author{
John Fryearson MB ChB MRCP and Dawn Adamson MRCP PhD \\ University Hospital of Coventry and Warwickshire - Cardiology, Clifford Bridge Road, Coventry CV2 2DX, UK
}

\begin{abstract}
Summary: A 25-year-old-woman presented to health-care services recurrently due to pleuritic chest pain. This increased during the first trimester of her first pregnancy. She was subsequently referred to a tertiary cardiology centre where she was seen by a cardiologist with a special interest in obstetric cardiology. After further investigations, an unusual cause of her pain was identified. Her investigations, management and her clinical course are presented here.
\end{abstract}

Keywords: cardiology, thoracic medicine, neoplasm

\section{CASE REPORT}

A 25-year-old primigravada was seen eight weeks into her pregnancy in the obstetric cardiology clinic of a tertiary cardiology centre. She had been admitted to her local hospital twice in the preceding three months with sharp pleuritic type chest pain localized to the left side and mammary region. It was aggravated by coughing, sneezing, yawning and could often last for hours. It was quite severe and debilitating at times and had required strong opioids to control the pain. The pain was beginning to increase in frequency and severity, though was still sporadic in occurrence, and it was this that prompted referral to the clinic.

Her previous investigations had demonstrated a rounded and prominent left heart border with some minor left basal haziness thought to be compatible with infection. A chest X-ray was repeated and again illustrated increased cardiothoracic ratio. An echocardiogram was requested to assess heart size, structure and function. Apart from mild thickening of the mitral valve, of no clinical significance, the heart itself was normal. However, there was a sizeable fluid filled mass seen abutting the left ventricular wall, which appeared to be cystic in nature and extracardiac (see Figure 1).

An urgent magnetic resonance imaging of the thorax was obtained and this demonstrated a large cystic mass occupying the space of the lower and midzone of the left thorax, measuring 10.6 by 8.7 by $13.4 \mathrm{~cm}$. There were a few thick-walled locules within the cyst, with mixed densities within these locules. There was mediastinal shift to the right, with a small pleural effusion and a normal hemidiaphragm (see Figure 2). The most likely diagnosis was felt to be a dermoid cyst, although a pericardial or bronchogenic cyst were less likely differentials.

The patient developed increasing pain requiring further admission to hospital and long-term continuation of opioid analgesia. In discussion with a thoracic surgeon, it was felt that surgical exploration and possible removal would be in

Correspondence to: John Fryearson

Email: dr_john_fryearson@hotmail.com the patient's and fetus's best interests to confirm the diagnosis, improve lung capacity throughout the rest of pregnancy and labour and reduce pain. An MDT discussion took place involving the cardiologist, surgeon and obstetrician and it was felt best to perform surgery in the second trimester. At this stage the fetal development would have been complete and there was a reduced risk of preterm labour compared with later pregnancy. It was also unclear whether this may be a hormonesensitive mass, which may have grown further in pregnancy and increased respiratory embarrassment already contributed to by a gravid uterus.

At 14 weeks our patient underwent surgery under general anaesthesia and thoracic epidural cover. A bronchoscopic examination was performed first which was normal. Then a muscle-sparing thoracotomy incision at the left fifth intercostal space was performed, which revealed a large mass medial to the lung tissue abutting the heart. There were no adhesions and no pericardial or pleural effusion identified. Using both blunt and sharp dissection, the phrenic nerve was isolated and preserved and the mass removed in one piece. Histological examination revealed a cystic nodule $15 \mathrm{~cm} \times$ $2 \mathrm{~cm} \times 7 \mathrm{~cm}$, which was unilocular containing greenish brown fluid. It had mixed extradermal, mesodermal and endodermal components, as well as a small amount of thymus tissue and was identified as a benign mature cystic teratoma. The patient did well after surgery and was discharged home a few days later without complication. She was reviewed at around 20 weeks and was found to be well, with her breathing feeling improved and improvement in her pain.

She went on to have an uncomplicated spontaneous vaginal delivery at term and gave birth to a healthy girl weighing $3350 \mathrm{~g}$.

\section{DISCUSSION}

Teratomas are a class of non-seminomatous germ cell tumour, tumours that develop from pluripotent embryonal or germ cells. They characteristically comprise cells from all three embryonic tissue types. They most commonly occur around the mid-line. Around $8 \%$ of all teratomas occur in the 


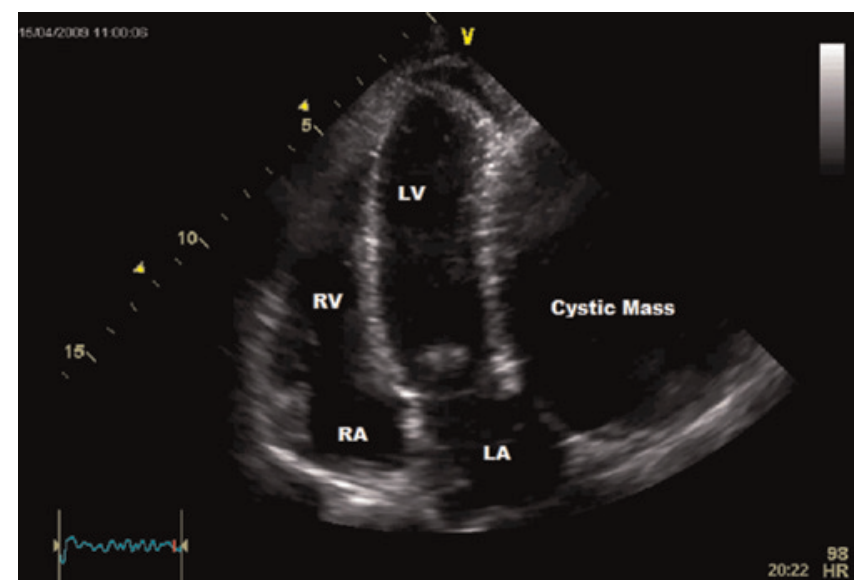

Figure 1 An apical four-chamber view showing the cystic structure adjacent to the left ventricular wall. LV, left ventricle; LA left atrium; RV, right ventricle; RA, right atrium

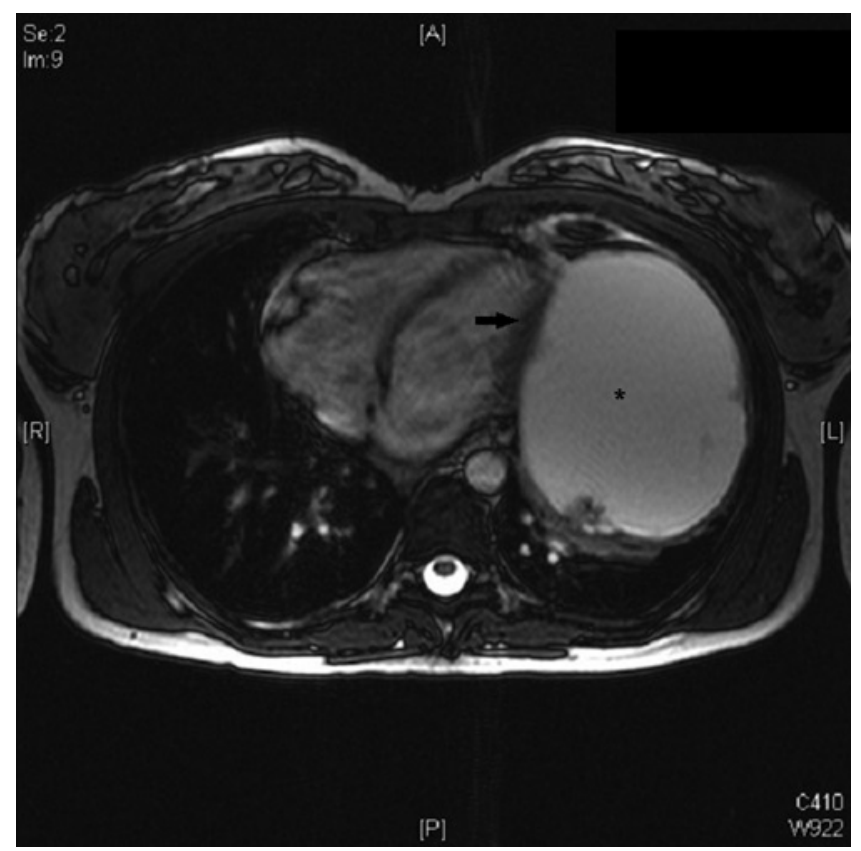

Figure 2 A T2-weighted magnetic resonance imaging image of the thorax showing the cystic structure occupying the majority of the left hemithorax in this plane and measuring approximately $10 \mathrm{~cm}$ by $8 \mathrm{~cm}$. "Denotes cystic mass and arrow shows mass abutting left ventricular wall

mediastinum, with a similar proportion of all mediastinal tumours being due to a teratoma. ${ }^{1}$ Mature (benign) teratomas occur with equal frequency in men and women typically in the third decade, while malignant teratomas occur predominantly in men. ${ }^{2}$ Presenting symptoms are dependent on size, with increasing respiratory embarrassment being caused by larger tumours, but include chest pain, cough as well as dyspnoea. A number of tumours are found incidentally in asymptomatic patients. In large tumours, occasionally erosion of the cystic capsule by enzymatic juices can cause rupture into lung parenchyma. Presentation with productive cough, which may be of sebum or hair (trichoptysis) is a pathognomonic, but exceedingly rare, sign of ruptured mediastinal teratoma. ${ }^{2}$ Management of all teratomas is usually with surgical excision, both to reduce the likelihood of growth and increasing compressive symptoms, and also to remove the small risk of malignant transformation. ${ }^{3}$

This case illustrates the pitfalls we all face when managing patients with rather non-specific symptoms. However, it also underpins the importance of taking seriously a patient who presents recurrently with the same problem. The pregnancy in this patient's case allowed her to be seen by a cardiologist earlier than perhaps she otherwise would have. Had she not been pregnant diagnosis may have been further delayed. This patient's symptoms worsened in pregnancy, and while there are other factors related to the gravid abdomen that may have been exacerbating this, it is known that pregnancy can cause an increase in the size of teratomas. ${ }^{4}$ The aetiology is not entirely clear but it is hypothesized to be due to oestrogen and progesterone sensitivity of the tumour itself. Ultimately, this patient's main problem was of debilitating pain, which on some occasions had required admissions for patient controlled analgesia. It seems likely that the pain was caused by compressive effects on the lung parenchyma and pleural layers.

This case demonstrates the difficulty in managing a pregnant patient who presents with respiratory symptoms - had she not presented in early pregnancy symptoms of dyspnoea may have been misinterpreted as being due the physiological and mechanical consequences of pregnancy itself. This is a problem seen frequently in obstetric cardiology with many patients presenting later due to misinterpretation of symptoms and signs. This patient required a multidisciplinary approach to her management, and given the complexity of the problem it would have been difficult to appropriately manage her any other way.

\section{DECLARATIONS}

Competing interests: None.

Ethical approval: N/A.

Guarantor: DA.

Contributorship: JF wrote the first draft of the manuscript. DA conceived the idea. Both authors reviewed and edited the manuscript.

Acknowledgments: We would like to thank the Echocardiography Department at University Hospital of Coventry and Warwickshire.

\section{REFERENCES}

1 Lewis BD, Hurt RD, Payne WS, Farrow GM, Knapp RH, Muhm JR. Benign teratomas of the mediastinum. J Thorac Cardiovasc Surg 1983;86:727-31

2 Strollo DC, Rosado de Christenson ML, Jett JR. Primary mediastinal tumors. Part 1. Chest 1997;112:511-22

3 Duwe BV, Sterman DH, Musani AI. Tumors of the mediastinum. Chest 2005;128:2893-909

4 Donnadieu AC, Deffieux X, Le Ray C, Mordefroid M, Frydman R, Fernandez $\mathrm{H}$. Unusual fast-growing ovarian cystic teratoma during pregnancy presenting with intracystic fat 'floating balls' appearance. Fertil Steril 2006;86:1758-9 